\title{
Performance Evaluation of Geocomposite Reinforcement in Flexible Pavements using Marshall Test Protocol
}

\author{
Adnan Qadir ${ }^{1, *}$ and Karam-un-Nisa Choudhary ${ }^{2}$ \\ ${ }^{1}$ Chairman, Department of Urban and Infrastructure Engineering, NED University of Engineering and \\ Technology, Karachi, Sindh, Pakistan \\ ${ }^{2}$ Graduate Research Assistant, Department of Urban and Infrastructure Engineering, NED University \\ of Engineering and Technology, Karachi, Sindh, Pakistan
}

\begin{abstract}
Asphalt concrete pavements are prone to cracking due to external loads and extreme weathering conditions. Generally the failed asphalt concrete is over laid with new asphalt concrete surface to improve the riding surface; however after sometime the crack reappears in the form of reflective cracking. Studies have found that such cracks can be controlled by introduction of Geo-synthetics layer before overlaying. The presented study presents one such effort in which the performance of such modification was evaluated by testing a number of modified specimens using Marshall's test protocol. The resulting Stability flow quotient at optimum asphalt content showed no appreciable improvement as expected. Hence it can be concluded that the performance of Geocomposite modified asphalt concrete cannot be evaluated using Marshall Test protocols.
\end{abstract}

\section{Introduction}

Asphalt pavement are designed to resist repetitive load from traffic without failure, under the prevailing climatic conditions. However, distresses do occur in the pavement and the conventional method of preventing distresses in to overlay it with asphalt concrete [1]. Sometimes this approach also fails to stop cracks from reoccurrence, thus making it undesirable for the agencies responsible for highway maintenance.

Reflective cracking is the one of common type of distress usually found on a newly overlaid asphalt concrete pavement. At present there is no acceptable technique of rehabilitation which can prevent the reflective cracking. Reinforcement of asphalt concrete with materials like fibers, polymers and Geocomposite have been found to be a reliable alternative option. Much research has been undertaken to ascertain that using of geosynthetics on failed pavement before overlaying can reduce reflective cracking [8]. The procedure involving the use of Geocomposite materials in pavements has been in use since 1960's. Geocomposite materials include geogrids, geocells, geomembranes, geotextiles and erosion control materials [2]. The reinforcement of asphalt concrete by Geocomposite layer is beneficial since it reduces the rutting susceptibility and improves the fatigue life $[3,4,5]$. It is found that the use of geotextile helps in sustaining the rut depth to a tolerable limit [6,

\footnotetext{
Corresponding author: cuid@neduet.edu.pk
} 
7]. The benefits of using Geocomposite in the pavements cannot be denied, however, the effectiveness of such modification needs to be tested under local traffic loading and weather conditions in a laboratory before it can be recommended to be used in full-scale pavements.

The presented study investigated the use of geotextile materials as underlay layer in asphalt concrete for reducing the reflective cracking. The primary reason for this study is to improve the deteriorating conditions of roads and make it sustainable for longer periods. The actual study itself have many segments, of which the findings of preliminary results is being presented in this conference of high repute.

\section{Objective and scope}

The presented study had an objective to investigate the influence of geo-composite as a reinforced layer in pavements by checking its stability-flow ratio. The objective was achieved by performance of standard AASHTO T 245-82 test (resistance to plastic flow asphalt concrete using Marshall Test apparatus) on the laboratory prepared specimens (controlled and geo composites). The presented findings are being limited to evaluation of the performance of Geocomposite material by using Marshall Test protocol only.

\section{Study hypotheses}

It was envisaged that the average stability flow value of modified sample would be higher as compared to similar relations for controlled samples.

\section{Materials and methodology}

\subsection{Materials}

Locally available material was used to prepare asphalt concrete samples in the study in accordance with local specifications (National Highways, NHA). The measured properties of the materials are being discussed separately in the relevant sections.

\subsubsection{Aggregate}

Aggregates are the most important ingredient in asphalt concrete, constituting $90-95 \%$ by weight. Fortunately, enough the locally available aggregates were found very much suitable and confirms to the local specifications. Hence selection of aggregate was not a difficult task in the study. These measured properties are indexed in Table 1.

The gradation of aggregates in asphalt concrete plays vital role in sustaining the traffic loads and extreme climatic conditions. The study used gradations in accordance with NHA standards. Figure 1 illustrates graphical view of the gradations incorporated in the study.

Table 1. Properties of Aggregates

\begin{tabular}{|c|c|c|c|}
\hline S.no & Standards & Description & Values \\
\hline $\mathbf{1}$ & BS-812 part 110 & Impact value & $20 \%$ \\
\hline $\mathbf{2}$ & BS 812 part 112 & Crushing value & $24 \%$ \\
\hline $\mathbf{3}$ & BS 812 part 105 & Flakiness & $18 \%$ \\
\hline $\mathbf{4}$ & BS 812 part 105 & Elongation & $10 \%$ \\
\hline $\mathbf{5}$ & AASHTO T96-83 & L.A. Abrasion & $28 \%$ \\
\hline $\mathbf{6}$ & AASHTO T85-81 \& T 228 & Bulk Specific gravity & 2.655 \\
\hline
\end{tabular}




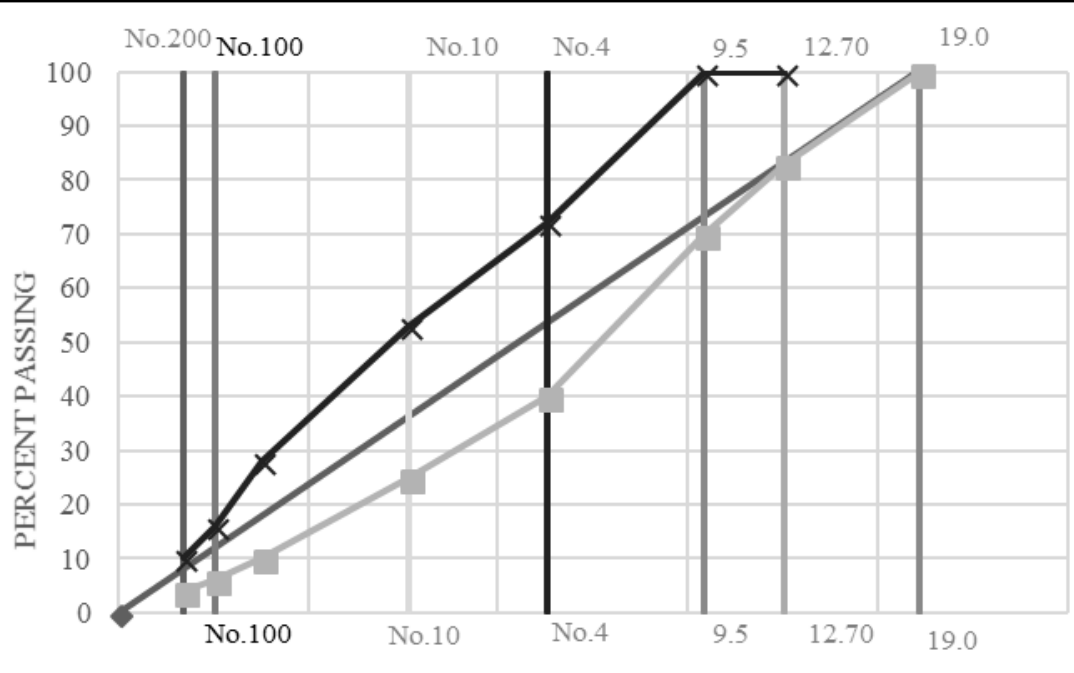

SIEVE SIZES

$\leadsto$ MAXIMUM DENSITY LINE

Fig.1. Aggregate gradation chart

\subsubsection{Asphalt}

The study used asphalt having penetration grade $60-70$ as per prevailing local practice. The other measure properties of asphalt are being presented in Table 2 .

Table 2.Test on Asphalt

\begin{tabular}{|c|c|c|c|}
\hline S. No & Standard & Description & Value \\
\hline 1 & AASHTO T49 & Penetration test & $6-7 \mathrm{~mm}$ \\
\hline 2 & AASHTO T53 & Softening point test & $42^{\circ} \mathrm{C}$ \\
\hline 3 & AASHTO T48 & Flash \& fire point test & $182^{\circ} \mathrm{C}, 222^{\circ} \mathrm{C}$ \\
\hline 4 & AASHTO T228 & Specific gravity test & 1.035 \\
\hline 5 & AASHTO T44 & Solubility test & $99.2 \%$ \\
\hline
\end{tabular}

\subsubsection{Geocomposite}

Geocomposite are polymeric products available in much form such as geotextile, geogrid, geonet, geomembranes, Geocomposite, clay liners, geofoam, geocells and geo-composites. The geotextile used in the study (Figure 2) is categorized as non-woven geogrid consisting of polyester and glass fiber material. The geogrids were equally spaced in both lateral and longitudinal directions. Since the testing facilities were not existent at the study area, it is assumed that the material properties provided by the local vendor are genuine. Table 3 presents the properties related to geocomposite used in the study as supplied by the local contractor 
Table 3. Properties of Geocomposite

\begin{tabular}{|c|c|c|}
\hline S. No & Description & Value \\
\hline 1 & Category & fiber glass, geo-grid composite geotextile \\
\hline 2 & Thickness & $2 \mathrm{~mm}$ \\
\hline 3 & crack intensity longitudinal & $30 \mathrm{KN} / \mathrm{m}$ \\
\hline 4 & crack intensity cross sectional & $30 \mathrm{KN} / \mathrm{m}$ \\
\hline 5 & break elongation ratio $\%$ longitudinal & Greater than or equal to 4 \\
\hline 6 & Mesh size & $12 \mathrm{~mm} \times 50 \mathrm{~mm}$ \\
\hline
\end{tabular}

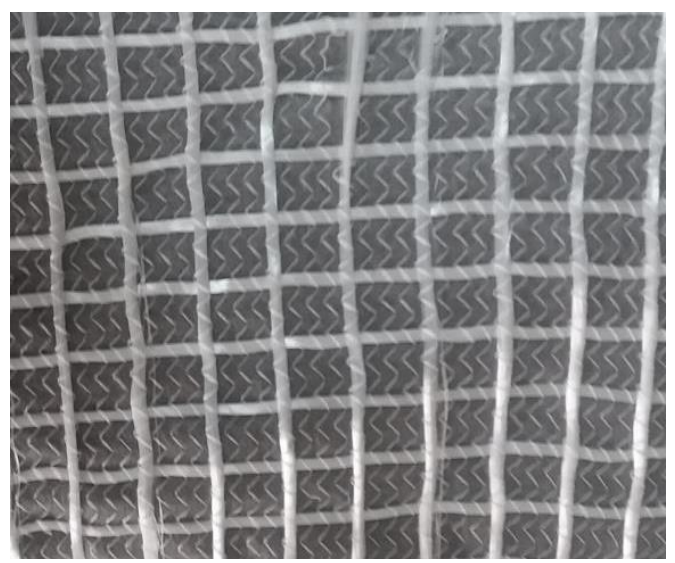

Fig.2. Fiber Glass Geo-Grid Composite Geotextile

\subsection{Methodology}

Following were the steps adopted for conducting the presented study

1.Trial sample preparation (including compaction).

2.Testing for Stability and flow.

3.Determination of optimum asphalt content (OAC).

4.Preparation of controlled and modified samples.

\subsubsection{Sample preparation}

Trial test specimens for both gradation (coarse and fine) were prepared using 3.5\%, 4\%, $4.5 \%, 5 \%, 5.5 \%$ asphalt content respectively. After mixing, sample was placed in the oven at $150^{\circ} \mathrm{C}$ for four hours (to induce short time aging), before compaction. Each of the samples was compacted with seventy five numbers of blows on each side. Samples were also prepared without compaction to determine maximum specific gravity of mixes according to test protocol of AASHTO T 209. These samples were needed to compare with compacted sample to calculate percentage air voids. The bulk density of each compacted sample ( according to AASHTO T 275) were determined by first weighting the sample in air and in water and then drying the surface to get its weight for saturated dry condition. Table 4 presents summary of test specimen prepared in the study. 


\subsubsection{Determination of OAC}

Once the entire test conducted the OAC were determined for each type of gradation using Asphalt Institute manual series (MS-2). In the present study $4.2 \%$ and $4.5 \%$ were confirmed as OAC. It is to be noted here that OAC required for fine gradation is more in comparison to OAC required for coarse gradation which make sense, since fine gradation have more surface area than coarse gradation. Ten confirmation samples were further prepared and tested to verify the validity of the OAC as determined.

\subsubsection{Preparation of controlled and modified samples}

Next three Samples were prepared without geotextile referred to as "control sample" and three more samples were prepared by incorporation of geotextile referred as "modified sample". Both of these samples were prepared with two type of gradation (coarse and fine) which was acquired from National Highway Authority (figure 1). To prepare modified samples following techniques were adopted.

-A disk of paper greased was placed inside the mould. Approximately half quantity of the prepared mix was poured into the mold and was mixed thoroughly with heated spatula and tamped 15 times around the parameter and 10 times in the interior until surface was smoothed out.

-A layer of Geocomposite was then inserted and the remaining mixture of asphalt mix were introduced in the mold, again tamping and filling the voids manually. Once

- again, the entire prepared specimen was tested for stability and flow test in Marshall Testing Machine. Fig. 3 illustrates the prepared samples.

Table 4. Summary of test specimen prepared

\begin{tabular}{|c|c|c|c|}
\hline & Description & \multicolumn{2}{|c|}{ Explanations } \\
\hline 1 & Aggregate Type & \multicolumn{2}{|c|}{ Limestone } \\
\hline 2 & Asphalt Type & \multicolumn{2}{|c|}{ Penetration grade $60-70$} \\
\hline 3 & Gradation & \multicolumn{2}{|c|}{ Coarse $(\mathrm{C})$ and Fine $(\mathrm{F})$} \\
\hline 4 & Air void content & \multicolumn{2}{|c|}{5.5 percent } \\
\hline 5 & Types of sample prepared & Loose & Compacted \\
\hline 6 & $\begin{array}{c}\text { Total number of samples with } \\
\text { replicates }\end{array}$ & $10(\mathrm{C})+10(\mathrm{~F})$ & $15(\mathrm{C})+15(\mathrm{~F})$ \\
\hline 7 & Sample size & - & $\begin{array}{l}10 \mathrm{~cm} \text { diameter; } \\
2.5 \mathrm{~cm} \text { height }\end{array}$ \\
\hline 8 & Shape of specimen & - & Cylindrical \\
\hline 9 & Test Conducted & Stability flow & $\begin{array}{l}\text { Maximum specific } \\
\text { gravity }\end{array}$ \\
\hline
\end{tabular}




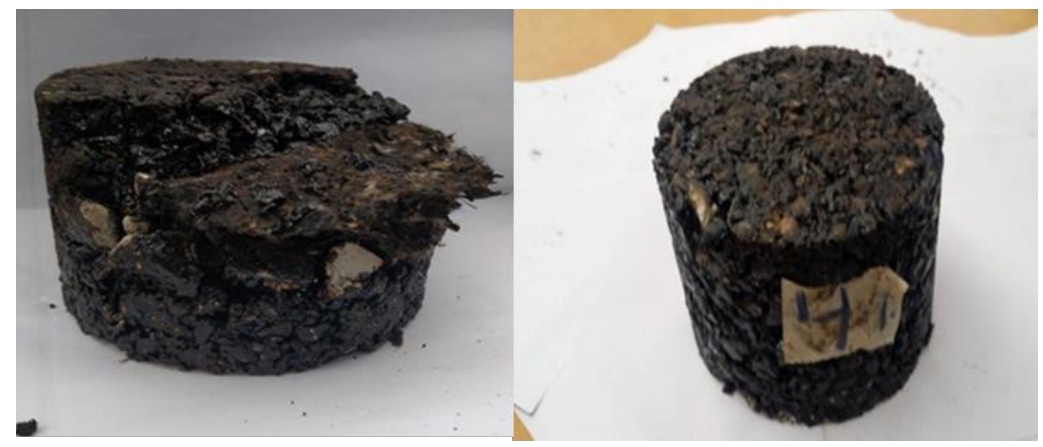

Fig. 3. (Left) Modified Sample, (Right) Control Sample

\section{Results and Discussion}

In this research Marshall Test Protocol was used to check the effectiveness of Geocomposite to improve the stability of flexible pavements. The study hypothesis assumed that the Geocomposite will increase the stability and strengthens the asphalt concrete. Total six samples were prepared to study the study hypothesis. These specimens were prepared according to the standard test specification AASHTO T 245-82 while Geo-composite was sandwiched between two layers of compacted asphalt concrete. The results are being illustrated in Fig. 4, 5\&6.

The result showed that coarse gradation showed better stability flow quotient as compared to fine gradation which is logical since the coarser mix are helpful in sustaining the compressive load (stability) as compared to fine graded mix. The results are identical with modified specimen made with coarse gradation (Figure 4).

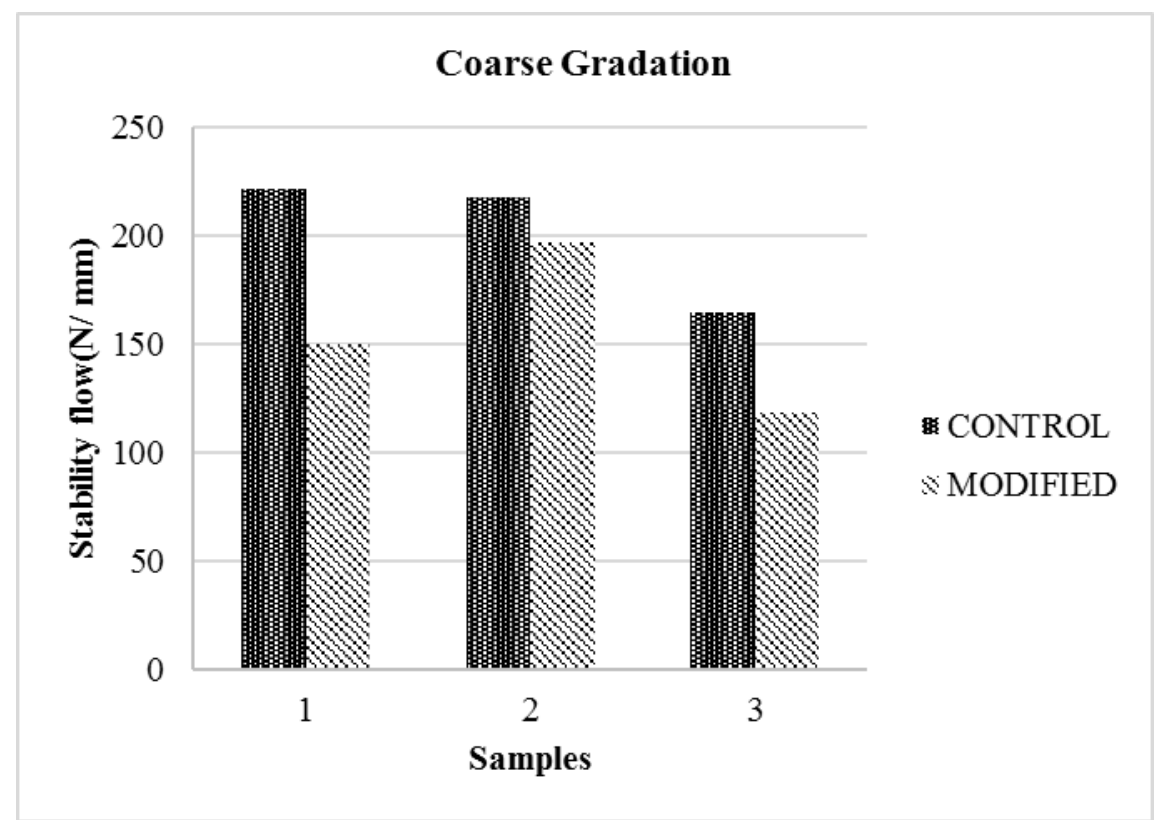

Fig. 4. Stability flow value for coarse gradation 


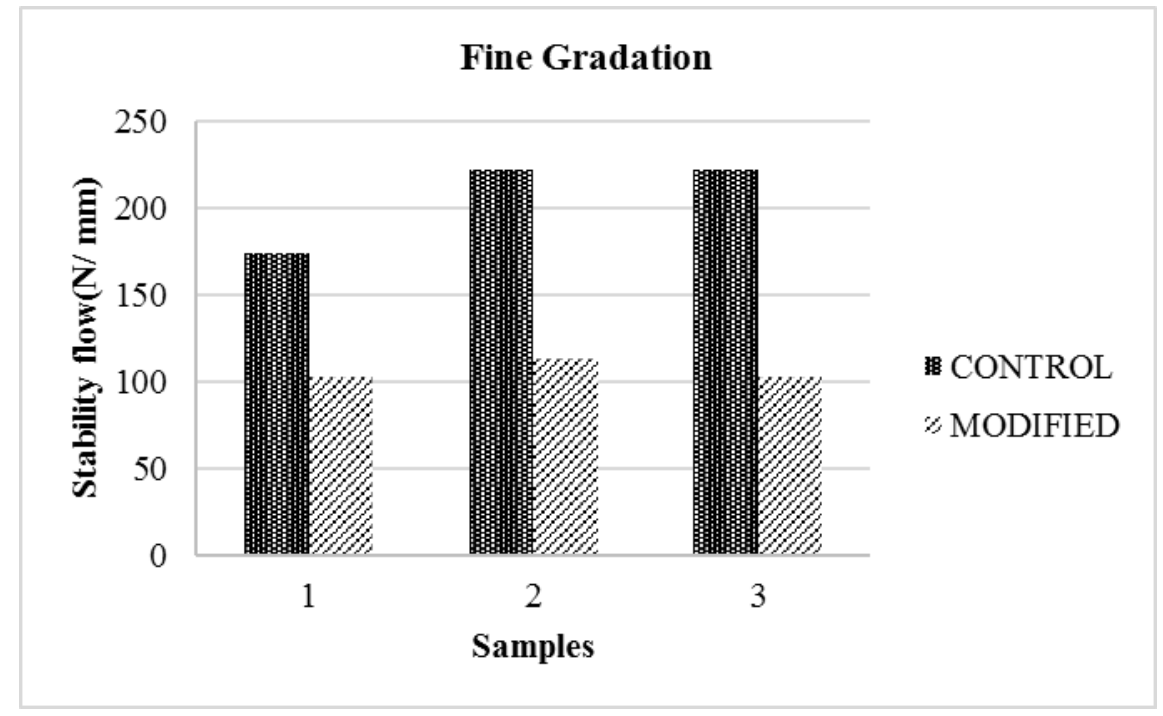

Fig. 3. Stability flow value for fine gradation

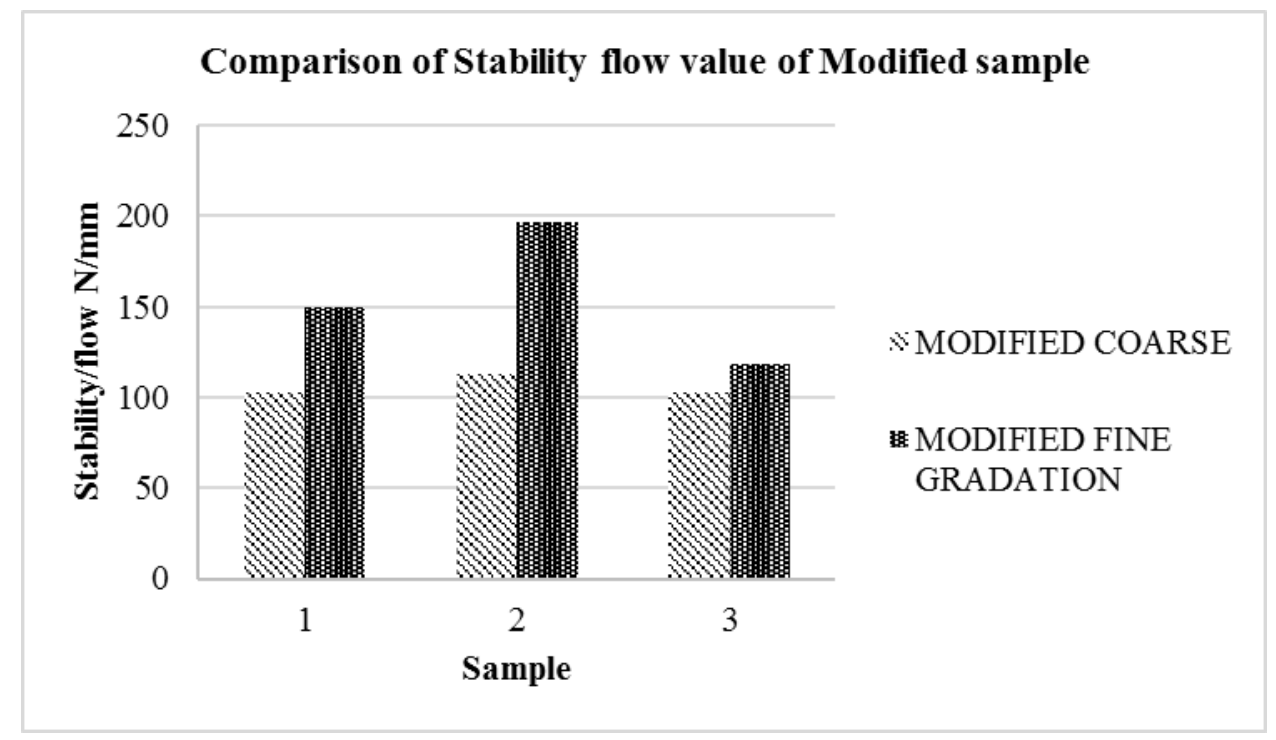

Fig. 6. Comparison of stability flow value of modified samples

From the results it can also be inferred that the incorporation of Geocomposite in the asphalt concrete did not improve the stability-flow quotient as was hypothesized in the study. This is primarily due to the following reasons.

1.The testing protocols of Marshal testing method in which the samples are tested cylindrically hence the contribution of the reinforcement cannot be properly evaluated. The sample may have failed due to splitting, rather to compressive force as expected (Fig. 7). 


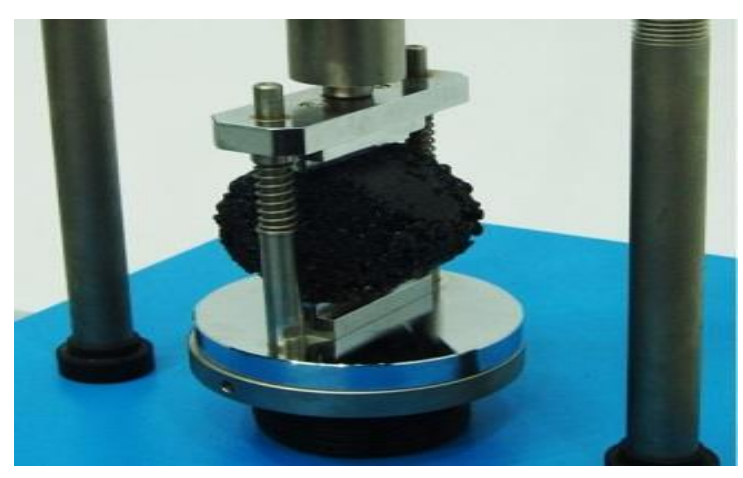

Fig. 7. Sample testing using Marshal Machine

2.During the test of stability and flow marshal test machine is design in such a way that the applied force is perpendicular to the specimen. Therefore, compaction has been done when the specimen is horizontal and stability is checked when the specimen is placed vertical. This contradiction would result the failure of geo-synthetic to increase the stability of asphalt concrete.

3.The failure may be due to inadequate bonding between geo-composite and asphalt concrete, since normally a test specimen is prepared in three layers, compacting manually and then through rammer, however in order to introduce geo composite, the manual compaction could not be done and also a sample was introduced in two layers.

While introducing geo-composite, a binder is needed on both side of material so that the material is bonded to the layers which were not possible during sample preparation with the present test setup.

\section{Conclusion and Recommendation}

From the experimental investigation carried out on the asphalt concrete specimens, it can be concluded that the geo-synthetic (Fiber glass geogrid composite geotextile) used in this study was not effective in controlling the cracks through Marshal Test protocol. It can also be concluded that Marshall Test is incapable of measuring such modification in asphalt concrete samples due its test protocols. Thus, the hypothesis that Geocomposite will improve the effectiveness of asphalt concrete through marshal test protocol is being rejected here.

Based on the results of this study it is recommended that Marshall Test should not be performed for testing such modification.

The study presented in this research paper is a part of research project titled "Investigation of the Rutting and Fatigue Performance of Asphalt Concrete Reinforced with Geotextile Materials" supported financially under National Research Program for Universities (NRPU) by Higher Education Commission Pakistan (HEC) at NED University of Engineering and Technology. The support is duly acknowledged here by the authors.

The author also acknowledges and thanks the administration of NED University, for sponsoring the trip for presenting the paper 


\section{References}

1. M. Baghee, K. Rehan, M. Abdelaziz, A Review on Fatigue and Rutting Performance of Asphalt Mixes, Essays, 6, 4, 670-682 (2011)

2. B. Lance, Geosynthetics Materials what are they? How are they used? Soils Factsheet, British Columbia, Ministry of Agriculture and Lands, Order No. 644.000-1 (2006)

3. M. Mirzapour, K. Rehan, M. Abdelaziz, K. Ali, An overview on the Use of Geosynthetics in Pavement Structures, Scientific Research and Essays, 6, 11, 22512258 (2011)

4. Viswanadham S and Satkalmi V, Field Trials with Polypropylene Woven Geotextiles, The First Pan American Geosynthetics Conference \& Exhibition, Cancun, Mexico (2008)

5. H.R.A. Hosseini, A.K. Darban, K. Fakhri, The Effect of Geosynthetics Reinforcement on the Damage Propagation Rate of Asphalt Pavements, Sharif University of Technology ScienticaIranica, 16, 1, 26-32 (2006)

6. K. Meccai, H. Eyad, Geotextiles in Transportation Applications, Second Gulf Conference on Roads, Abu Dhabi (2004)

7. E. Pasquini, M. Pasetto, F. Canestrari, Geocomposite against Reflective Cracking in Asphalt Pavements: Laboratory Simulation of a field Application, Road Materials and pavement Design, 16, 4, 815-835 (2015) 